\title{
Design and Flight Test Results of Robust Attitude Controller for the ETS-VIII Spacecraft
}

\author{
Tomoyuki Nagashio* Takashi Kida* Takashi Ohtani ${ }^{* *}$ \\ Yoshiro Hamada** \\ * University of Electro-Communications, Chofu, Tokyo \\ ** Japan Aerospace Exploration Agency, Chofu, Tokyo
}

\begin{abstract}
This paper reports the flight test results of robust attitude controller for Engineering Test Satellite VIII (ETS-VIII) spacecraft. The main mission of ETS-VIII is to support nextgeneration mobile digital communications, and for this purpose it is equipped with two large deployable antenna reflectors and a pair of large solar paddles that rotate around the pitch axis. For the spacecraft, the authors have proposed and demonstrated several control methods based on the advanced control theories for the linear parameter varying multi-input multioutput system. The aim is to develop a baseline for future controller design technology for this class of satellite instead of existing classical PID control laws. This paper describes the on-orbit experiment results and compares these controllers performance.
\end{abstract}

Keywords: Large flexible spacecraft, Linear parameter varying system, Symmetric controller, Two degrees-of-freedom control, Gain-scheduling control, $\mu$-synthesis

\section{INTRODUCTION}

Recently, there is a trend that spacecraft is equipped with large flexible antenna reflectors and solar paddles to enhance communication performance and electric power to meet mission requirements. Controlling such large flexible spacecraft with high accuracy requires control of structural vibrations as well as attitude. In view of this, many control schemes have been studied up to now (e.g., Drai and Bordier 1996; Somov et al. 2007), and some on-orbit control experiment results have been reported (e.g., Irwin et al. 1995). The main common goal of these has been to guarantee robust stability against both higher vibration modes and modal parameter uncertainties.

With the same goal, an on-orbit attitude control experiment using Engineering Test Satellite VI (ETS-VI) was carried out in 1994 (Kida et al. 1997) in which several types of $H_{\infty}$ dynamic output feedback controllers were designed and their effectiveness was demonstrated. These controllers were three single-input single-output (SISO) linear time invariant (LTI) controllers designed for a spacecraft model described in a paddle-fixed reference frame.

Based on this experience, a robust control on-orbit experiment has been planned to be carried out on Engineering Test Satellite VIII (ETS-VIII), which was launched into geosynchronous orbit in December 18, 2006 using H-IIA launch vehicle No. 11 by Japan Aerospace Exploration Agency. ETS-VIII is the largest satellite that Japan has developed to date, with a size of $40 \times 37 \mathrm{~m}$ and a mass of $3000 \mathrm{~kg}$ (Fig. 1). Since the spacecraft has two large deployable reflectors and two solar paddles that rotate around the pitch axis, the dynamics has coupling between the three axes, and the system parameters change according to the paddle rotation. Therefore, the attitude control system

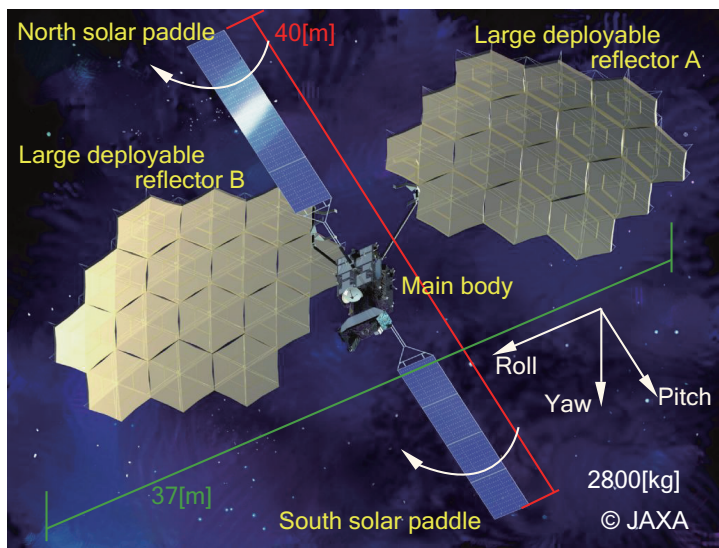

Fig. 1. A view of ETS-VIII.

must guarantee robust stability against above mentioned higher vibration modes and modal parameter uncertainties for a linear parameter varying (LPV), multi-input multioutput (MIMO) system. From this viewpoint, the authors have proposed several control methods (Hamada et al. 2007, Ohtani et al. 2009, Nagashio and Kida 2009, Nagashio et al. 2010), by applying advanced control theories in order to develop a baseline for future controller design technology for this class of satellite instead of existing classical PID control laws. This paper reports the on-orbit experiment results using them.

\section{SPACECRAFT SYSTEM AND DYNAMICS MODEL}

The main mission of ETS-VIII is to develop mobile communication technologies in the next generation. For the mission, it has two large deployable reflectors (LDR) with a size of $17 \times 19 \mathrm{~m}$ in the roll axis direction. Additionally, a 
pair of large solar array paddles (PDL) with a size of $19 \times 2$ $\mathrm{m}$ are appended in the pitch axis direction for supplying the electric power. The PDL rotate around the pitch axis at the rate of $360 \mathrm{deg} /$ day so that they continually face the sun. The three-axis attitude control subsystem (ACS) of ETS-VIII is composed of earth sensors assembly (ESA), inertial reference unit (IRU), rate integrated gyro assembly (RIGA) and four reaction wheels (RW) generating \pm 0.04 Nm maximum torque. The on-board computer of ACS operates at $16 \mathrm{~Hz}$, and the attitude angles and their rates are estimated by using these sensors and a determination logic based on the Kalman filter. In the operation, the required attitude accuracy of the roll and the pitch are $\pm 0.05 \mathrm{deg}$, that of the yaw is $\pm 0.15 \mathrm{deg}$. The data of attitude angles, attitude rates, control torque commands and so on are monitored through the house keeping telemetry at $0.5 \mathrm{~Hz}$. Besides the RW, this spacecraft carries reaction control system (RCS) which consists of an apogee kick engine, twelve bi-propellant thrusters and four ion engines.

Through the analysis of numerical simulations, the dynamical equation of ETS-VIII is given by the following hybrid equations which are composed of the rigid main body rotation and the flexible appendages vibration.

$$
\begin{aligned}
& J(\delta) \ddot{\theta}+\sum_{i} \Delta_{i}(\delta) \ddot{\eta}_{i}=u+w \\
& \Delta_{i}^{T}(\delta) \ddot{\theta}+\ddot{\eta}_{i}+2 \zeta_{i} \Omega_{i} \dot{\eta}_{i}+\Omega_{i}^{2} \eta_{i}=0
\end{aligned}
$$

where $i=n, s, a, b$ denotes the north/south PDL and the $\mathrm{a} / \mathrm{b} \mathrm{LDR}, \delta \in \mathbb{R}$ is the paddle rotation angle, $\theta \in \mathbb{R}^{3}$ is the attitude angle, $u \in \mathbb{R}^{3}, w \in \mathbb{R}^{3}$ are the three-axis torque control input and disturbance input. The disturbance $w$ is assumed to have the bounded $L_{2}$ norm because the main source is the orbit control thruster firing. $\eta_{i} \in \mathbb{R}^{n_{i}}$ is the modal coordinate of the $i$ th appendage. The inertia matrix $J(\delta)$ and coupling matrix $\Delta_{i}(\delta)$ vary depending on the PDL angle $\delta . \Omega_{i}^{2}=\operatorname{diag}\left[\omega_{i 1}^{2}, \cdots, \omega_{i n}^{2}\right]$ is a modal stiffness matrix where $\omega_{i j} \in \mathbb{R}$, and $\zeta_{i} \in \mathbb{R}$ denote modal frequency and damping ratio. By a finite element method (FEM) analysis in a cantilevered condition, the modal frequency, the coupling matrices and modal parameters of eight modes for each appendage have been obtained. Thus, the obtained full-order model has $n=3+n_{n}+n_{s}+$ $n_{a}+n_{b}=35$ coordinates.

For the attitude control, the estimates of the attitude angles and their rates are available as the measurement outputs

$$
y_{d}=\theta, \quad y_{v}=\dot{\theta}
$$

The spacecraft equations (1) and (2) can be compactly described as follows:

$$
\begin{aligned}
& M(\delta) \ddot{p}+D \dot{p}+K p=L u+L w \\
& y_{d}=L^{T} p, \quad y_{v}=L^{T} \dot{p}, \quad y=\left[\begin{array}{ll}
y_{d}^{T} & y_{v}^{T}
\end{array}\right]^{T}
\end{aligned}
$$

where $p=\left[\begin{array}{lllll}\theta^{T} & \eta_{n}^{T} & \eta_{s}^{T} & \eta_{a}^{T} & \eta_{a}^{T}\end{array}\right]^{T} \in \mathbb{R}^{n}$. It is noted that the second order differential equation (3) has two features: firstly

$$
M(\delta)>0, D \geq 0, K \geq 0, \forall \delta
$$

holds from the modal identity (Likins 1970) and secondly, the system (3) is stabilizable and detectable since the rank conditions
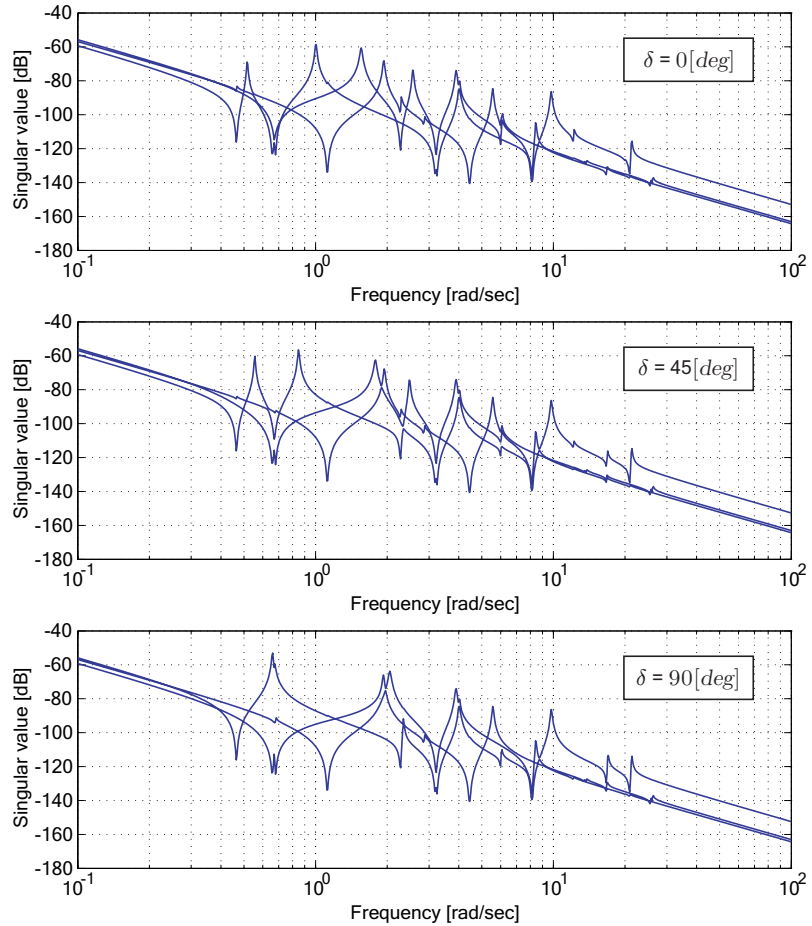

Fig. 2. Singular value plots of the ETS-VIII open-loop system for $\delta=0$ (top), 45 (middle) and $90 \mathrm{deg}$ (bottom).

$$
\operatorname{rank}[K, L]=\operatorname{rank}[D, L]=n
$$

are satisfied for all $\delta$ (Ikeda et al. 1993).

The dependency of the spacecraft model on the PDL angle $\delta$ is evaluated by singular value plots of the open-loop plant from the control input $u$ to the measurement output $y_{d}$ for the PDL angles of $\delta=0,45,90 \mathrm{deg}$ (Fig. 2). It is apparent that many vibration modes exist in the lower frequency range from $0.06 \mathrm{~Hz}$ to $4.0 \mathrm{~Hz}$ and they are exchanged with each other according to the PDL angle. Therefore, the spacecraft must be treated as a LPV MIMO system.

\section{ON-ORBIT EXPERIMENT}

The ETS-VIII has two typical control requirements: an attitude tracking capability to follow a reference step command for antenna calibration, and a disturbance attenuation capability to the orbit control thruster firing for precise pointing of the antennas. They are common requirements to the future typical spacecraft. Therefore, the authors have made the on-orbit experiment plan which consists of one case of attitude angle step response and four cases of disturbance responses caused by the RW, the east-west station-keeping thrusters and the attitude maneuver thrusters. The details are described with the on-orbit experiment results in section 6 .

\section{CONTROLLER DESIGN}

The controller design objective is to obtain a reduced order controller that robustly stabilizes the LPV MIMO spacecraft against the unstructured uncertainties of higher vibration modes and the structured uncertainties caused by the inaccuracy of modal identification before launch. 


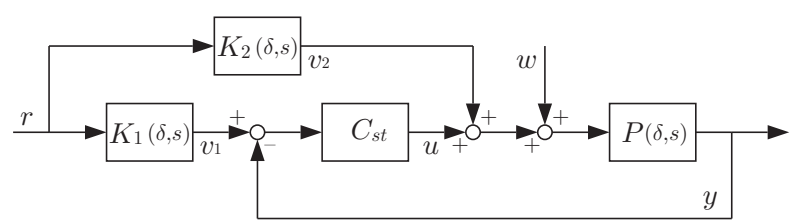

Fig. 3. Block diagram of two degrees-of-freedom control system.

Additionally, it must satisfy the trade-off control specifications of the disturbance attenuation and attitude tracking capabilities. In order to satisfy these requirements, the authors have designed four types of controllers by applying the robust control theory for the LPV MIMO system:

(1) Gain scheduling control law using linear interpolation (GS).

(2) Linear time invariant control law by applying $\mu$ synthesis $(\mathrm{Mu})$.

(3) Two degrees-of-freedom control law based on symmetric dynamic output feedback controller (2DOFDynamic).

(4) Two degrees-of-freedom control law based on symmetric static output feedback controller (2DOFStatic)

Additionally, in order to compare the performance of the proposed controllers, a simple standard PD feedback controller has been also designed. It has almost the same capability as the bass controller which is used for the main mission of ETS-VIII.

Among them, this paper describes the design method for the two degrees-of-freedom control system using the symmetric static output feedback controller ${ }^{1}$. This control system consists of the feedback control part for the stabilization and the feedforward control part for the reference tracking (Fig. 3). The feedback controller, which is static output feedback using the measured outputs $y_{d}, y_{v}$, is known to guarantee the internal stability of the closedloop system and the robustness against the model errors based only on the non-parametric structure condition by preserveing symmetric properties (Balas 1979, Arbel and Gupta 1981). In our experiment, the controller is optimized for the disturbance attenuation by minimizing the $\mathrm{H}_{\infty}$ norm of the closed-loop system. On the other hand, the feedforward controller is designed as a gain scheduling controller by applying a model matching method to the LPV system (Apkarian et al. 1995, Nagashio et al. 2010). In order to optimize each controller for all $\delta$, we utilize the fact that $M(\delta)$ is bounded for all $\delta$ and given by the convex decomposition as

$$
M(\delta)=\sum_{i=1}^{\sigma} a_{i} M_{i}, M_{i}>0, \quad a_{i} \geq 0, \quad \sum_{i=1}^{\sigma} a_{i}=1 .
$$

The each controller property is summarized below.

\subsection{Static Output Feedback Controller}

First, let us consider a static output feedback controller

1 Other controller design methods have been described in the references (Hamada et al. 2007, Ohtani et al. 2009, Nagashio and Kida 2009).

$$
u=-C_{d} y_{d}-C_{v} y_{v}=-C_{s t} y
$$

where

$$
C_{d}=C_{d}^{T}>0, C_{v}=C_{v}^{T}>0 .
$$

By using the controller to the plant (3), the closed-loop system can be described as

$$
M(\delta) \ddot{p}+D^{*} \dot{p}+K^{*} p=L w
$$

where

$$
K^{*}=K+L C_{d} L^{T}>0, \quad D^{*}=D+L C_{v} L^{T}>0 .
$$

Then, it is known that the closed-loop system (9) always guarantees the non-parametric internal stability based only on the positive-definite symmetric conditions (4) and $(10)$ when the disturbance $w=0$. Therefore, the stability is robust even for the LPV system having the uncertainties of higher vibration modes and model errors. For the controller design, the authors consider to optimize the disturbance attenuation capabilities for the controlled output $z=y_{d}=L^{T} p$ in the framework of $\mathrm{H}_{\infty}$ controller synthesis as follows.

$$
\left\|L^{T}\left(s^{2} M(\delta)+s D^{*}+K^{*}\right) L\right\|_{\infty}<\gamma, \quad \forall \delta
$$

where $\gamma>0$ is the $\mathrm{H}_{\infty}$ norm of the closed-loop system. However, it is also known that the bounded real lemma (BRL) for the static output feedback $\mathrm{H}_{\infty}$ controller synthesis is given by a bilinear matrix inequality, that is not efficiently solved. To cope with this difficulty, the authors have proposed the design method by giving a structure to the solution of BRL (Nagashio et al. 2010). Then, the design condition becomes simultaneous LMIs

$$
\begin{aligned}
& -Q_{i}+\frac{1}{\gamma} S S^{T}<0, \quad i=1,2, \cdots \sigma \\
& C_{d}>0, \quad C_{v}>0, \quad \gamma>0
\end{aligned}
$$

where

$$
Q_{i}=\left[\begin{array}{cc}
2 \alpha K^{*} & \alpha D^{*} \\
\alpha D^{*} & 2 D^{*}-2 \alpha M_{i}
\end{array}\right], S=\left[\begin{array}{cc}
\alpha L & L \\
L & 0
\end{array}\right] .
$$

Therefore, by applying the convex optimization algorithm for minimizing the common $\gamma$, the controller (7) can be obtained. In (13), $\alpha>0$ is an arbitrary design parameter. It is noted that a low-pass filter is added to the angular velocity signal $y_{v}$ in order to attenuate measurement noise of the RIGA.

\subsection{Feedforward Controller}

Next, in order to improve attitude maneuver performance to the reference signal $r \in \mathbb{R}^{3}$, a feedforward controller is designed by a model matching method. The design condition is given by minimizing $\gamma_{g}>0$ such that

$$
\left\|G_{r}-G_{y r}\right\|_{\infty}<\gamma_{g}, \forall \delta
$$

where $z_{r}=G_{r}(s) r$ denotes a given constant transfer function matrix of the reference model, $z_{g}=G_{y r}(\delta, s) r$ is the closed-loop system which consists of the plant, the feedforward controller and the above feedback controller, and $z_{g}=y_{d}$. For the precise model matching of the LPV system, the authors design the feedforward controller having two parts $K_{1}(\delta, s)$ and $K_{2}(\delta, s)$ by modifying the gain scheduling $\mathrm{H}_{\infty}$ controller synthesis (Apkarian et al. 
1995). The details of design method and the theoretical viewpoint are found in the previous paper (Nagashio et al. 2010). It is noted that this feedforward controller functions only for the non-zero step command.

\section{GROUND VERIFICATION}

Before the on-orbit experiments, a static closed-loop test (SCLT) on the ground has been carried out for verification of the implementation issues. The SCLT setup consists of the engineering model of ACS on-board computer, the telemetry command simulator and the spacecraft dynamics simulator. It operates in the real-time as the actual onorbit experiment. Through the test, it has been confirmed that the ACS functions well and each controller achieves performances expected in the simulation of all experiment cases. As the results of SCLT, the required memory size and the processing time of the ACS on-board computer for the each control law are compared in Table 1.

Table 1. Comparison of controllers

\begin{tabular}{|c|c|c|}
\hline & Memory size [byte] & Processing time [msec] \\
\hline GS & 4140 & 1.40 \\
\hline $\mathrm{Mu}$ & 7948 & 2.28 \\
\hline 2DOF-Dynamic & 14220 & 4.26 \\
\hline 2DOF-Static & 14780 & 4.77 \\
\hline PD & 668 & 0.22 \\
\hline
\end{tabular}

\section{ON-ORBIT EXPERIMENT RESULT}

Based on the above pre-launch evaluation, the on-orbit experiments have been carried out during two periods: June 21-29 in 2009, and February 28 - March 7 in 2010. The data of each experiment case have been obtained twice so that the PDL angles have different initial values to verify the control performance as the LPV system. The extracted experiment results are shown in Figs. 4 - 11.

Among them, Fig. 4 shows the attitude angles of the step response in the first experiment, Fig. 5 is the enlargement of the pitch axis result in this case. Additionally, Fig. 6 is the second experiment results of the step response, and Fig. 7 is the enlargement of the roll axis. On the other hand, Figs. 8 and 10 show the results of the rectangular disturbance responses caused by the RW in the first and second experiments, Figs. 9 and 11 are the enlargements of the roll and the pitch axes respectively. These data have been obtained through the house keeping telemetry. We have compared the obtained results with the SCLT and have confirmed they closely resemble. The features of the flight results are summarized below.

\section{- Step response}

In this case, the reference attitude angle commands are given as \pm 0.05 deg for the roll and pitch axes individually. The proposed controllers have achieved shorter settling time than the PD controller. Since the results of first and second experiments are also the same, it is confirmed that the proposed controllers are effective to the LPV system caused by the PDL rotation. Both of the two degreesof-freedom controllers have achieved same results on all axes in the first and second experiments. This is because the model-matching design method works well. The gain scheduling controller has achieved the same performance
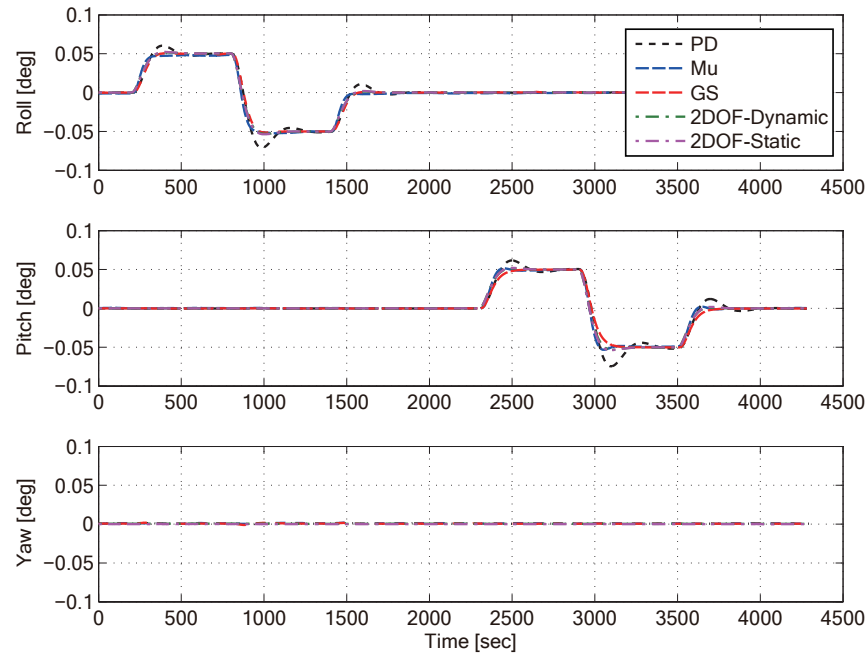

Fig. 4. Time history of roll, pitch, yaw attitude angle responses to step command of first experiment.

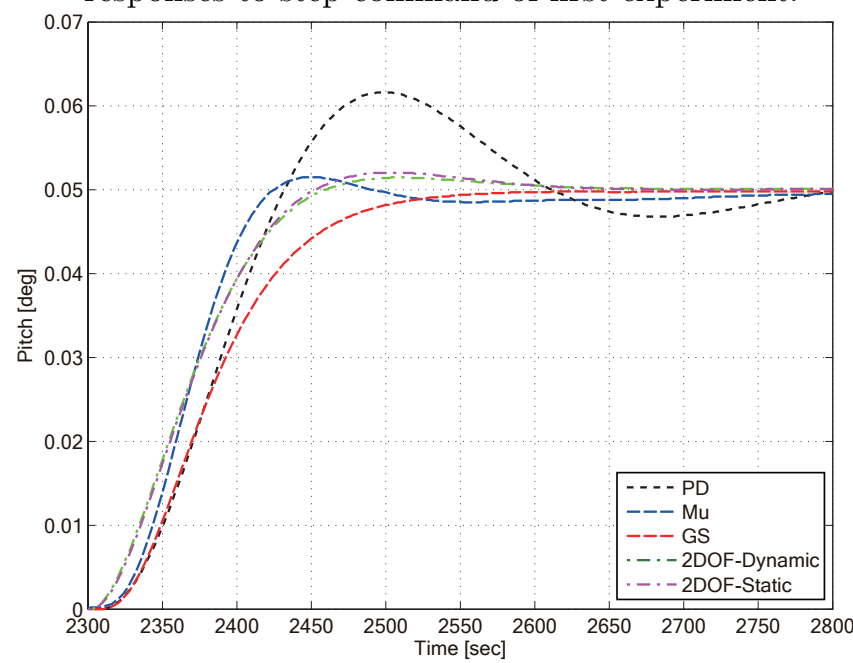

Fig. 5. Time history of pitch attitude angle responses to step command of first experiment.

to the parameter change by the PDL rotation, while the results of rise time, settling time and overshoot are different in each axis. It depends on the modal vibration direction which is included in the reduced order plant for the controller design. In almost all cases, $\mu$-synthesis has achieved shortest rise time in the proposed controllers although the settling time is the longest. The reason is supposed to be that the order of the controller is higher than others.

- Rectangular disturbance response

In this case, the disturbances caused by the RW are added as the rectangular torque $0.04 \mathrm{Nm}$ for $30 \mathrm{sec}$ to each axis individually. From the results, it is apparent that the proposed controllers are effective in reducing the influence of disturbances compared with the PD controller, as well as they are robustly stable against the PDL rotation. The performances of the maximum amplitude and the settling time using each controller are shown to have the tendency same as the step responses.

As described so far, the proposed controllers have achieved expected performances in almost all experiment cases. 

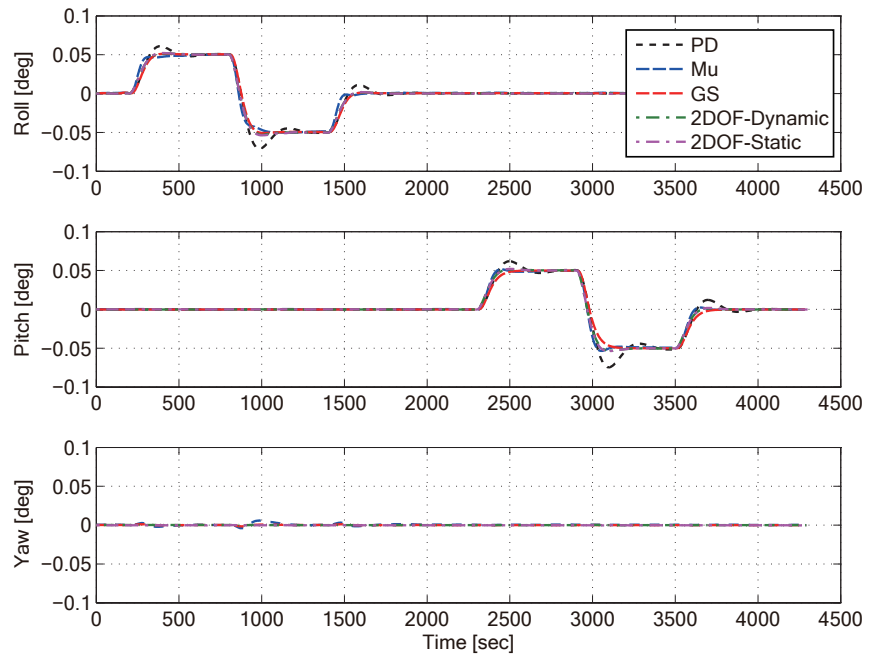

Fig. 6. Time history of roll, pitch, yaw attitude angle responses to step command of second experiment.

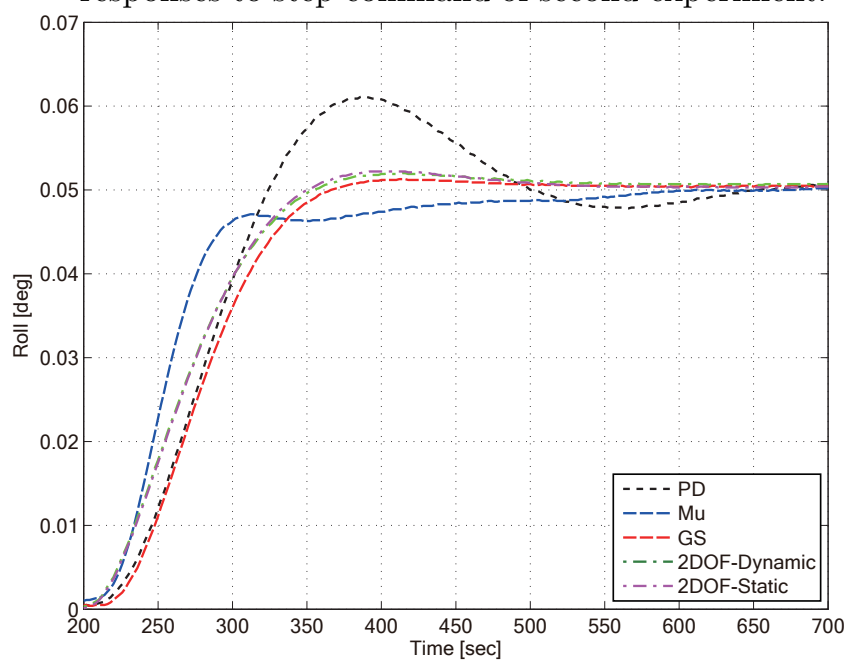

Fig. 7. Time history of roll angle responses to step command of second experiment.

However, the results of the impulse disturbance response caused by the east-west station-keeping thruster firing are different in the experiment and the SCLT. The both results of the two degrees-of-freedom controller with the symmetric static output feedback controller are compared in Fig. 12. The disturbances in this case are generated by the thruster firing of $625 \mathrm{msec}$, and the both thruster firing of the east and the west operate during $600 \mathrm{sec}$ interval. In the SCLT, the disturbance torques for the east direction have been assumed as $-0.3,-0.5,1.8 \mathrm{Nm}$ in the roll, pitch, yaw axes respectively, and the west are $-0.2,1.2,-1.8 \mathrm{Nm}$, through the numerical model analysis. By comparing the experiment and the SCLT, we find the discrepancy only in the pitch axis responses. The reason is presently concluded to be that the disturbance torque around the pitch axis has been changed from the prospected one since the center of mass has been changed by spending the fuel for the thruster. The further detailed analysis by using the results is currently under work.

Finally, the average of the settling time of step responses and the maximum amplitude ratio of all disturbance responses is shown in Table 2. The two degrees-of-freedom
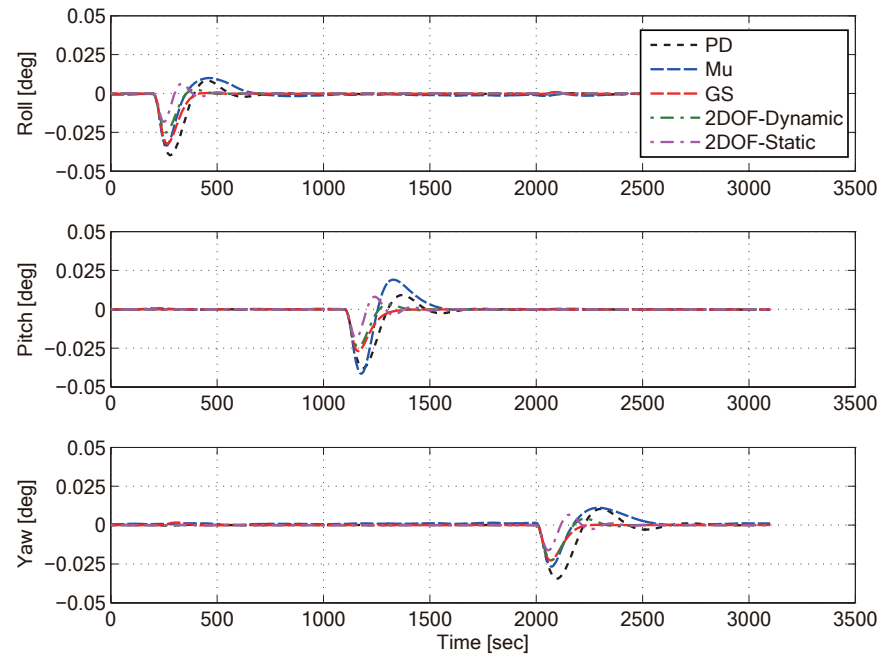

Fig. 8. Time history of roll, pitch, yaw angles in response to rectangular disturbance of first experiment.

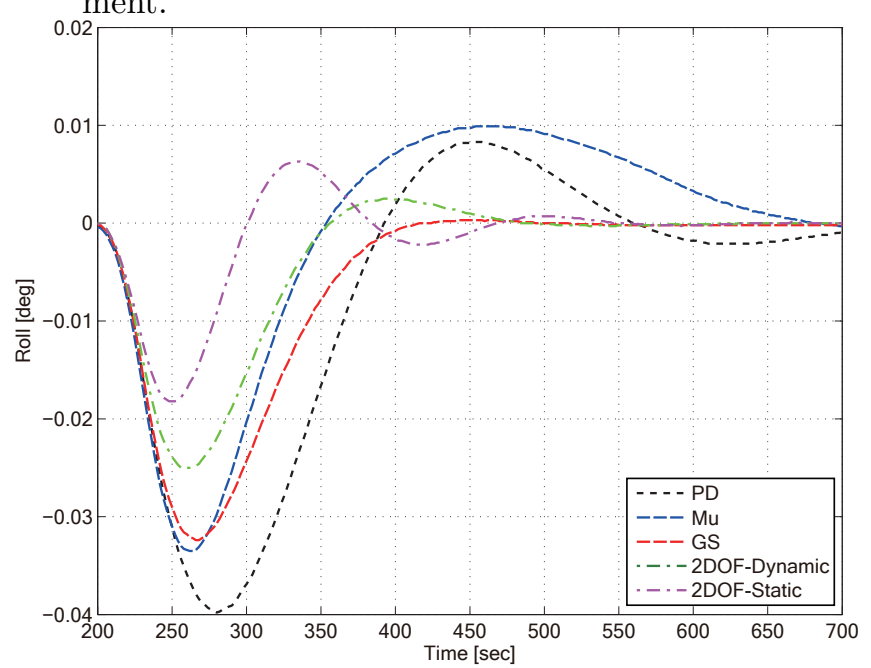

Fig. 9. Time history of roll angles in response to rectangular disturbance of first experiment.

controller with the symmetric static output feedback controller has achieved the best performance in both capabilities. This is because the optimization of control performance using the LMI has functioned more effectively compared with the other controller optimization, and the static type has the wide bandwidth. On the other hand, the gain scheduling controller has also achieved good performances under the lowest required memory size and the shortest processing time among the proposed control laws as in Table 1 . The $\mu$-synthesis controller works sufficiently well using the linear time invariant controller for LPV MIMO system.

Table 2. Average data of performance

\begin{tabular}{|c|c|c|}
\hline & Settling time [sec] & Max. amplitude [\%] \\
\hline GS & 144 & 82.5 \\
\hline $\mathrm{Mu}$ & 153 & 85.0 \\
\hline 2DOF-Dynamic & 130 & 67.5 \\
\hline 2DOF-Static & 126 & 51.4 \\
\hline PD & 250 & 100.0 \\
\hline
\end{tabular}



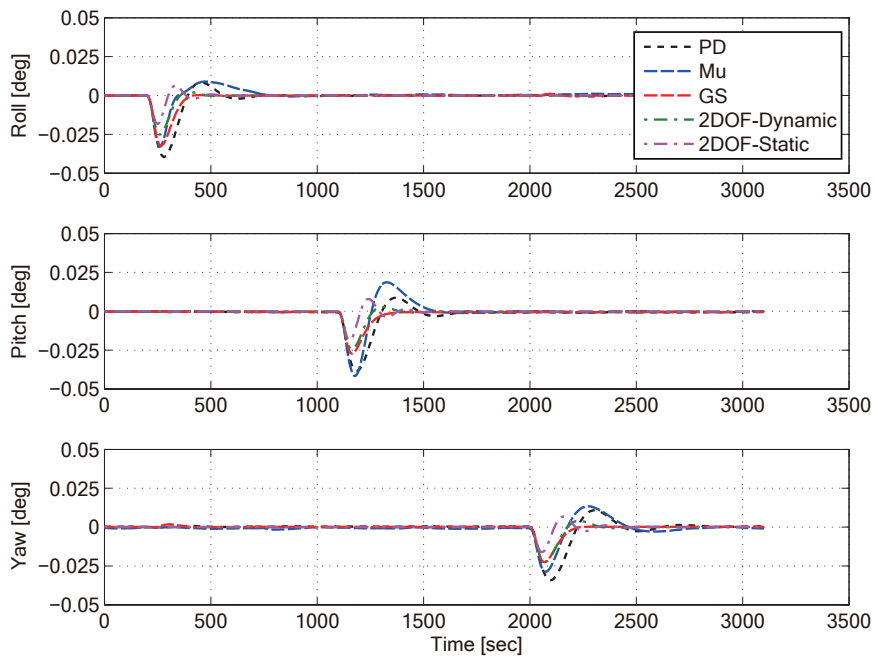

Fig. 10. Time history of roll, pitch, yaw attitude angles in response to rectangular disturbance of second experiment.

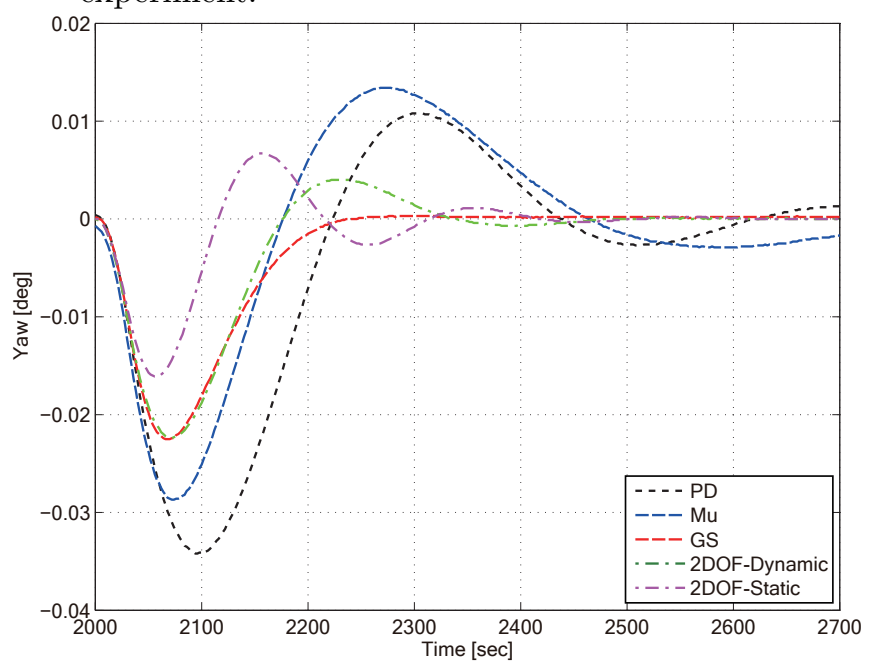

Fig. 11. Time history of yaw angles in response to rectangular disturbance of second experiment.

\section{CONCLUSION}

This paper has reported results of on-orbit attitude control experiment using ETS-VIII spacecraft. From the results, it has been confirmed that the proposed control systems are effective for the precise attitude control of the LPV MIMO spacecraft. Our current work is the detailed analysis to make clear the difference between the numerical simulation and the experiment. Difference of the on-orbit identified model and the pre-launch model by FEM analysis which is used for the controller design will be evaluated in the frequency domain as well as the time domain.

\section{REFERENCES}

Apkarian, P., Gahinet, P., and Becker, G. (1995). Selfscheduled $H_{\infty}$ control of linear parameter-varying systems. Automatica, 31, 1254-1261.

Arbel, A. and Gupta, N.K. (1981). Robust colocated control for large flexible space structures. J. of Guidance, Control, and Dynamics, 4, 480-486.

Balas, M.J. (1979). Direct velocity feedback control of large space structures. J. of Guidance, Control, and Dynamics, 2, 252-253.
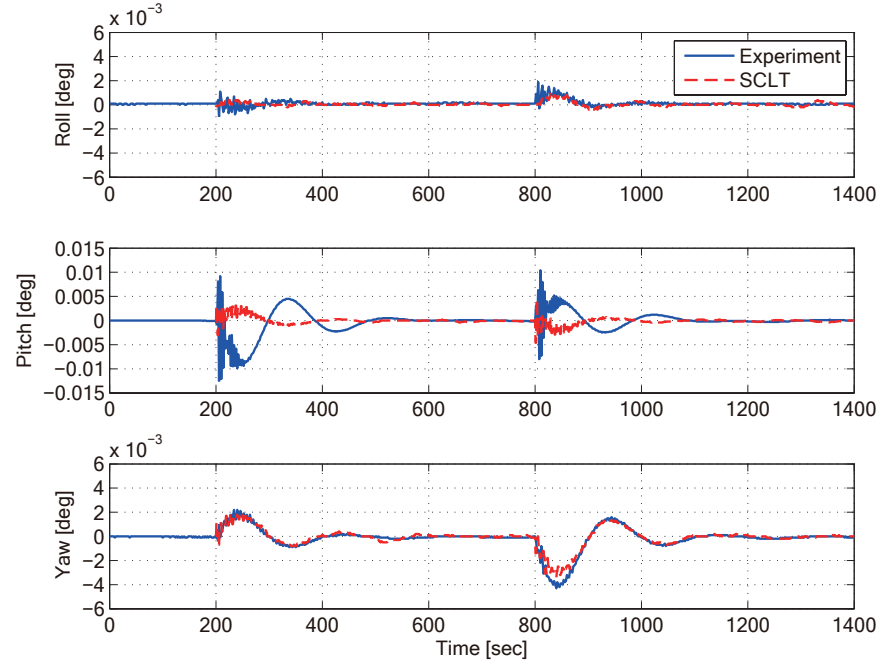

Fig. 12. Time history of roll, pitch, yaw attitude angles of experiment and SCLT in east-west impulse disturbance responses using 2DOF-Static.

Drai, R. and Bordier, M. (1996). Robust attitude control of a telecommunication satellite: a l.m.i. approach. Proc. of 3rd Inter. Conf. on Spacecraft Guidance, Navigation and Control Systems, 247-251.

Hamada, Y., Ohtani, T., Kida, T., and Nagashio, T. (2007). Gain scheduling controller design for engineering test satellite-viii. Proc. of 17th IFAC Symposium on Automatic Control in Aerospace, TH-01-01.

Ikeda, M., Koujitani, M., and Kida, T. (1993). Optimality of direct velocity and displacement feedback for large space structures with collocated sensors and actuators. 12th IFAC World Congress, VI, 91-94.

Irwin, R.D., Glenn, R.D., Frazier, W.G., and Lawrence, D.A. (1995). Analytically and numerically derived $h_{\infty}$ controller designs for hubble space telescope. J. of Guidance, Control, and Dynamics, 18, 214-221.

Kida, T., Yamaguchi, I., Chida, Y., and Sekiguchi, T. (1997). On-orbit robust control experiment of flexible spacecraft ETS-VI. J. of Guidance, Control, and Dynamics, 20, 865-872.

Likins, P. (1970). Dynamics and control of flexible space vehicles. JPL Technical report.

Nagashio, T. and Kida, T. (2009). Robust control of flexible mechanical systems by utilizing symmetry and its application to large space structures. IEEE Trans. Control Syst. Technol, 17, 671-680.

Nagashio, T., Kida, T., Ohtani, T., and Hamada, Y. (2010). Design and implementation of robust symmetric attitude controller for ets-viii spacecraft. Control Engineering Practice, 18, 1440-1451.

Ohtani, T., Hamada, Y., Nagashio, T., Kida, T., Mitani, S., Yamaguchi, I., Kasai, T., and Igawa, H. (2009). Robust attitude control using mu-synthesis for the large flexible satellite ets-viii. Journal of Space Technology and Science, 25-1, 27-40.

Somov, Y., Butyrin, S., Titov, G., and Yakimov, Y. (2007). Robust gyromoment spacecraft attitude control with precise pointing a flexible antenna. Proc. of 17th IFAC Symposium on Automatic Control in Aerospace, WE$04-1$. 